\title{
FILOSOFÍA DEL NACIMIENTO: APROXIMACIÓN DESDE LA FENOMENOLOGÍA CORPOREIZADA E INTERSUBJETIVA DE MERLEAU- PONTY
}

Philosophy of Birth: Approach from the Embodied and Intersubjective Phenomenology of MerleauPonty

\author{
Ronnie Videla Reyes \\ Universidad Central/ Universidad Santo Tomás, La Serena, Chile \\ ronnie.videla@ucentral.cl \\ Marco Cortez Burotto \\ Universidad de La Serena, La Serena, Chile \\ fil.educa@gmail.com
}

\section{Resumen}

El presente estudio aborda el nacimiento como problema filosófico desde una perspectiva intersubjetiva y corporeizada derivada del análisis del Cuerpo y el Otro presente en la Fenomenología de la Percepción de Merleau-Ponty. A partir de lo anterior, se discuten las limitaciones que tendría la coexistencia (Mitdasein) de Heidegger y la Otredad de Lévinas enmarcada en la relación del rostro en su intento de aproximarse a una filosofía del nacimiento desde una perspectiva de mundo compartido (madre-padre-hijo). Además, se plantea la necesidad de incorporar al problema filosófico sobre el nacimiento tres etapas: embarazo-nacimiento-puerperio, ya que estas son fundamentales para ilustrar de manera profunda la dimensión relacional y corporeizada de este proceso que exhibe temporalidad e historicidad.

Palabras clave: Filosofía del Nacimiento, Mitdasein, Otredad, Cuerpo, Intersubjetividad.

\begin{abstract}
The present study approaches birth as a philosophical problem from an intersubjective and embodied perspective derived from the analysis of the Body and the Other present in the Phenomenology of Perception of Merleau-Ponty. Based on the foregoing, the limitations of the Beingwith-the-other (Mitdasein) of Heidegger and the Otherness of Levinas framed in the relationship of the face in its attempt to approach a philosophy of birth from an approximation of shared world (mother-father-son). In addition, there is a need to incorporate the three stages of pregnancy into the philosophical problem: pregnancy-birth-puerperium, since these are fundamental to illustrate in a profound way the relational and embodied dimension of this process that exhibits temporality and historicity.
\end{abstract}

Keywords: Philosophy of Birth, Mitdasein, Otherness, Body, Intersubjectivity.

Fecha de Recepción: 25-06-2019 - Fecha de Aceptación: 22/07/2019 
Yo sé por dónde nace, de qué grietas exhala su destello. Como empieza a romperse. Con qué dulzura anunciase su gracia. Cuánto es el gran latido de su prudencia. Qué congoja la estremece al tocarme por adentro.

Gonzalo Rojas

\section{Introducción}

En el poema "La materia es mi madre" de Gonzalo Rojas, del cual se desprende el epígrafe de este texto, es posible asistir a la certeza fisiológica del cuerpo. A partir de éste, se impone la matriz originaria que es el lenguaje y la maleabilidad que exhibe el cuerpo del origen durante la fragilidad del movimiento de quien se anuncia. En este caso, la palabra. Si nos quedamos con el nacimiento en su dimensión relacional y encarnada de un cuerpo para el cuerpo, como es el caso de la madre que lleva consigo a su hijo y que interacciona con los otros, podemos identificar tres aspectos fundamentales que proporcionan el trasfondo espaciotemporal para la realización del espacio vital en el cual los seres humanos llegamos al mundo: embarazo-nacimiento-puerperio. Estas etapas proveerían una propuesta no antes planteada para problematizar filosóficamente el nacimiento desde el serpara-la-vida en contraposición al ser-para-la-muerte y el ser-contra-la-muerte.

En la fenomenología trascendental y existencialista se ha olvidado el nacimiento debido a que se ha priorizado el ser para la muerte (Diez 2012). El auge de la filosofía de la medicina en el siglo XX ha suscitado el planteamiento de nuevas preguntas acerca de la existencia humana, incorporando un giro en la reflexión hacia fenómenos ligados a la salud respecto a cómo se entiende el cuerpo, el dolor y el sufrimiento de quien padece una enfermedad. En otras palabras, el foco de cuestionamiento filosófico obedece hoy a las patologías existenciales que se derivan del deterioro de la vida $\mathrm{y}$, por lo tanto, de las erosiones que va consumando la existencia (Aurenque y Jarán 2018). A partir de esto, la muerte parece ser más importante que el nacimiento, ya que su trasfondo inexorable determina nuestra relación con el tiempo $\mathrm{y}$, por ende, resulta más atractivo reflexionar acerca de la hondura de la conciencia de la finitud, que la condición auspiciosa del cuerpo del ser humano en gestación o del neonato.

El nacimiento en la filosofía no es considerado un fenómeno existencial como lo es la muerte, por el contrario, parece ser un evento insustancial desprovisto de mayor reflexión. Sin embargo, el nacimiento nos confronta con un aspecto fundamental de la existencia como es el problema del origen. El nacimiento impone desde ya un ser-para-la-vida que ha sido relegado no sólo desde el genuino interés, sino también, desde el abandono de una comprensión intersubjetiva producto del predominio de visiones adscritas a la idea de una 
conciencia solipsista o a la lectura de la evidencia del otro como diferencia de lo trascendente. Una de las perspectivas solipsistas acerca del otro es la de Heidegger (1998), en cuya propuesta analítica existencial del Dasein, aborda tal problema como coexistencia o Mitdasein ${ }^{1}$. Se puede encontrar también el Mitwelt o mundo compartido para problematizar el modo en que el sí-mismo comparece con el otro. No obstante, este conjunto de propuestas resulta infructuoso para abordar el problema del otro en la filosofía del nacimiento. De la misma manera, el ser initium, propuesto por Arendt (1993) hace hincapié en el ser humano que desembarca en solitario durante el nacimiento y a que el lugar extraño al que llega, sólo se le presenta a través de la acción en el trasfondo del ser principium que es el mundo predeterminado.

Según los planteamientos de Lévinas (1995) en la aproximación a la otredad, éste arguye que lo otro es la negación del Ser. Esta negación se basa en la epifanía del rostro y la mirada que se distancian del lenguaje como representación solipsista del mundo dado que en la palabra como tema se contiene el otro. En relación a lo expuesto, es menester preguntarse: ¿es el nacimiento un fenómeno de poco interés para la filosofía, debido a que es considerado un aspecto singular de la experiencia? $\mathrm{O}$ ¿es de poco interés debido al suelo inmemorial del bebé que no permite analizar la posibilidad de mundo, ya que no hay vestigios de historicidad en la fuerza oculta de la matriz uterina que resuelva la ausencia de lenguaje? Sin duda, estas preguntas interpelan la escasez de reflexiones acerca del nacimiento como problema filosófico. La propuesta de este estudio recae en la comprensión de la filosofía del nacimiento desde una perspectiva corporeizada que denote la idea del nacimiento como un fenómeno intersubjetivo (Merleau-Ponty 1975).

En relación con este último aspecto, es menester colocar al nacimiento como problema existencial fuera de una lectura de lo otro en una tesis del ser como interioridad o exterioridad. Así como también abandonar la idea del nacimiento como singularidad de la presencia de un otro que está en vías de realización en la medida que actúa en el mundo. Por ello es necesario preguntarse: ¿qué lugar ocupa el hijo/a que lleva la madre consigo? ¿Es el nacimiento un acontecimiento desarticulado de una trama mayor que otorga condiciones para su realización, como es el proceso de embarazo y el puerperio? ¿Cuáles son las variaciones de la existencia que debiese considerar una filosofía del nacimiento como configuración relacional? Estas preguntas resultan necesarias para repensar en primer lugar a la filosofía del nacimiento como objeto de problematización. El énfasis de las preguntas expuestas, nos lleva a pensar en el lugar del otro durante el embarazo, al nacimiento y al puerperio como etapas que configuran historicidad y temporalidad a las variaciones de la existencia de quienes viven el proceso.

\footnotetext{
${ }^{1}$ En este contexto remitimos a la traducción de Ser y tiempo de Rivera (1997).
} 
En el presente artículo, sostenemos que el nacimiento debe ser considerado un fenómeno existencial de tipo intersubjetivo (Merleau-Ponty 1975), ya que corresponde a un fenómeno histórico del cual no se puede sustraer al individuo que llega al mundo en un espacio compartido con otros. De hecho, porque este evento mismo tiene significado para uno a través de los demás. A partir de lo anterior, y con el objetivo de formular una reflexión original en la filosofía del nacimiento planteamos lo siguiente:

i. El nacimiento no es un evento singular/transversal que consista solamente en la expulsión de un individuo, sino más bien, corresponde a un proceso histórico que involucra el embarazo y el puerperio. Para efectos de profundidad del análisis, en este texto solo haremos énfasis en el embarazo y el nacimiento.

ii. El embarazo es la etapa fundacional de la filosofía del nacimiento, ya que posibilita y opera como matriz ontológica de la existencia. Asimismo, el embarazo no es un hecho exclusivamente biológico que le sea propio a la madre, sino más bien, es un modo de vivencia relacional que incorpora variaciones de la existencia de quienes participan de este proceso.

iii. En el marco de la escasez de una comprensión profunda de lo otro para una aproximación intersubjetiva a la filosofía del nacimiento, el concepto de coexistencia tratado por Heidegger, presenta una limitación, dado que se deriva de la analítica del Dasein, por lo tanto, se centra en una interioridad. La lectura del Otro de Lévinas como superación del sujeto trascendental, la consideramos problemática para el tratamiento de la alteridad en la idea del Rostro como resonancia ética del ser como exterioridad. Planteamos más bien, la idea de una comprensión de lo otro intersubjetiva y corporeizada con independencia de una lectura solipsista y exterior del ser del otro. Para esto nos basamos en la tesis de Merleau-Ponty acerca del Cuerpo del Otro entendido como un Nosotros en el en-sí, cuyo aspecto de la interioridad o exterioridad, resultan ser las dos caras de un mismo acto.

La necesidad de preguntarnos por el ámbito fenoménico de la corporalidad humana responde al deseo de comprender cómo es que nosotros existimos corporalmente en un mundo. ¿De qué manera las variaciones del cuerpo obedecen a variaciones subjetivas sobre el embarazo? ¿Cómo irrumpe el otro como hijo/a en el embarazo para la madre y el padre? ¿De qué manera varían conjuntamente sus existencias?

\section{Coexistencia o Mitdasein en Heidegger}

Es importante mencionar que el ímpetu de emprender una reflexión sobre cómo el otro se constituye en el marco de una filosofía del nacimiento, obedece a criterios 
fundacionales de toda pregunta que pretenda interrogar la existencia humana. En el abordaje de la coexistencia se encuentra un conjunto de aspectos relativos a la comprensión que Heidegger (1998) hace del fenómeno del mundo, de la que se destacan tres perspectivas fundamentales: el mundo del sí mismo, el mundo circundante y el mundo compartido. El mundo del sí mismo como el mundo circundante, operan desde un "ser para la vida", compareciendo tanto el mundo de la vida como el propio despliegue del ser en el espacio. Lo que le otorga al modo de vivir la idea de mundo es la síntesis de un conjunto aspectos por la cual la vida se confronta fácticamente y adquiere significatividad. Considerando que la presencia de los otros surge en la facticidad, es que al ser le es dado ser. De este modo, el espacio vital en el cual la existencia se realiza desplegada temporalmente ocurre desde un trasfondo de articulación de sentido por el cual el mundo se "hace suyo" (Johnson 2012).

En una aproximación al fenómeno del mundo como manifestación de la vida humana con otros, cobra relevancia el mundo compartido. Para una lectura del mundo compartido, es necesario insistir en que la vía de reflexión surge desde la analítica del Dasein, pero con matices en la comprensión establecidos en el existir cotidiano. A partir de lo anterior, se propone que la comprensión del otro debe ser considerada como un sentido propio de la vivencia fáctica del otro y no como la aprehensión de un otro. La presencia del otro que se anuncia en la vivencia corresponde a otro Dasein que difiere de la vivencia misma, pero que se le es dado a partir de ella. Este modo de coexistencia surge en el existir cotidiano por el cual el mundo deviene en la vinculación de la vida con otras vidas, a lo que Heidegger denominó coexistencia.

Debido a la presencia de los otros que surge en la facticidad, es posible asumir que el otro es siempre un "Ser en relación a" (Johnson 2012 50). El modo de relación de comparecer con los otros obedece a un estar en la misma situación, ya que están ahí siendo co-partícipes del mundo. En otras palabras, desde el comparecer de los Dasein, se deriva el nosotros como una apertura del mundo circundante en el cual las acciones compartidas en una situación permiten el aparecer de los otros. La alteridad del otro no aparece independiente del contexto, ya que la vivencia de un nosotros se encuentra ligada al actuar de los otros que comparecen como instancia de un mundo compartido. Este mundo compartido donde hay un otro que asiste conmigo "no puede ser entendido fuera de aquello que no soy yo, por el contrario, viene a reafirmar la identidad que soy conmigo, y más aún, la comunidad mutua del existir" (Johnson 2012 53).

Considerando que la coexistencia surge del movimiento del estar siendo en el ahí, el cual se ha establecido de antemano como aquello que le es dado a ser, la ocupación del espacio vital en la cotidianidad de la existencia sitúa la concreción 
del otro como un "encuentro de antemano en el mundo". Dado que el Dasein es la apertura del ahí, que proyectado en su temporalidad se deja acaecer, el otro aparece producto de esta posibilidad de "tener que ser" en el espacio de lo posible. El otro desde esta perspectiva existencial comparece en un mundo que abierto, es ya compartible, como un estar dispuesto a ser-con-otros. Lo anterior refuerza la tesis de que el otro es proyección del ser como interioridad.

\section{La Otredad de Lévinas: el ser como exterioridad}

En respuesta crítica al sujeto trascendental, Lévinas toma distancia de lo que constituyó la enseñanza fundamental de Husserl, sobre todo en lo que se refiere a la epojé y a la reducción fenomenológica. El pensador lituano se da cuenta de que el otro no puede ser sometido a una reducción similar a la que se propuso frente al yo psicofísico, puesto que a diferencia de lo que sucede con el sujeto empírico, del cual cabe que se efectúe la correspondiente epojé, no puede hacerse lo mismo en el caso del otro (Lévinas 1995a). Incluso en un trabajo tan temprano como el Tiempo y el otro, parte no ya del ser, sino de la soledad del existente. Esta soledad existencial sólo puede ser conjurada a través del encuentro con el otro, que en ese momento es pensado como lo femenino (Lévinas 1993). El encuentro con el otro y la superación de la soledad se cumple en el eros. El tiempo al que se alude asume un sentido completamente distinto del que le otorga Heidegger en su analítica. Para el filósofo alemán, el tiempo es el horizonte de trascendencia del ser, puesto que la verdad como des-ocultamiento del ser acontece en el tiempo. Para Lévinas (1947), por el contrario, el tiempo es el otro, cuya presencia permite la trascendencia del sujeto, como un ser-contra-la-muerte y no para-la-muerte, como piensa Heidegger.

Lévinas profundiza en Totalidad e Infinito su planteamiento modificando algunas de sus tesis anteriores. El punto de partida se basa en la relación de un ente con otro ente, y no la diferencia ontológica entre ser y ente, como en Heidegger. Esta relación ya no pasa por el eros sino por el lenguaje. Lévinas entiende el lenguaje como una relación cuya característica fundamental es que sus miembros se absuelven mutuamente en el momento de entrar en relación. En este sentido, el ego del sí-mismo envuelve al otro. A esta relación, Lévinas le dará el nombre de ética, a la cual entenderá como separación y distinción (1995a). En relación a lo anterior, ¿cómo se produce este encuentro con el otro y qué puede significar de un modo más preciso? En esta obra a la que hacemos alusión ahora, Lévinas parte de una tesis: el ser es exterioridad. El encuentro con el otro es siempre traumático debido a su violencia fundamental. ¿Por qué esta violencia? Según Lévinas, es que el otro no sólo no es como nosotros, sino que la esencia de su otredad consiste precisamente en la alteridad (1993). 
Ahora, si la esencia de la otredad es alteridad, ¿cómo irrumpe el otro? Según Lévinas, el otro irrumpe mediante el cara-a-cara. Eso y sólo eso puede significar la ética. En el lenguaje, teníamos que ambos miembros de la relación, se absolvían mutuamente. Lo que Lévinas sostiene con fuerza, es que, en dicha relación, el otro no se encuentra al mismo nivel que el mismo, sino que la ética entraña una relación eminentemente asimétrica. En este sentido el 'mismo' que es el sujeto lo entiende como apologético. Este sujeto se ve confrontado cuando se tropieza con el otro. ¿Cómo sucede aquello? Por la interpelación a la que el otro lo somete. El otro interpela. ¿Cómo lo hace? Respuesta: a través del rostro. El rostro es la huella del infinito, o sea, un puro significante en ausencia del significado que le otorga su sentido (Lévinas 1995b). ¿Qué puede significar lo anterior? Lévinas plantea que esto se produce cuando la sincronía en la que se mueve la conciencia se ve perturbada por la diacronía en la que acontece la llamada del otro. El otro interpela desde un pasado inmemorial, y es este desfase temporal lo que puede alterar la paz de la conciencia, la cual se ve en la obligación de responder ante una acusación de la que ignora su procedencia, pero de la que no consigue escapar (Lévinas 1995b).

\section{La filosofía del nacimiento desde una aproximación corporeizada e intersubjetiva}

No podemos permanecer en esta alternativa de no comprender nada acerca del sujeto o de no comprender nada acerca del objeto. Es preciso que encontremos el origen del objeto en el corazón mismo de nuestra experiencia, que describamos la aparición del ser y comprendamos cómo, de forma paradójica, hay para nosotros un en-sí (Merleau-Ponty 1975 89).

A diferencia de los planteamientos anteriores centrados en la comprensión de lo otro como interioridad en Heidegger (1998) cuando refiere a que el otro "no puede ser entendido fuera de aquello que no soy yo" y la idea de Lévinas (1995) acerca del Otro como exterioridad, en el cual, sólo es posible referir a éste desde una relación ética que se resiste al radio del yo-lenguaje, ya que se impone el rostro como epifanía que fija la categoría de infinito, proponemos en términos de Merleau-Ponty (1975) que el otro es a su vez aquello que resulta del encuentro de lo interior-exterior, es decir, que la percepción exterior y la percepción del propio cuerpo varían conjuntamente porque son las dos caras de un mismo acto. En este sentido, estamos siendo en la percepción, por lo tanto, el otro que se presenta ante mí, adquiere alteridad a partir del encuentro en que mi cuerpo se encuentra, debido a que "la posición precisa del punto tocado será la invariante de los sentimientos diversos que del mismo tengo según la orientación de mis miembros y mi cuerpo, el acto de atención puede fijar y objetivar esta invariante porque se ha 
situado a distancia respecto de los cambios de la apariencia" (Merleau-Ponty 1975 49).

A partir de esta cita, aquello que comparece como otro es siempre una reversión del encuentro del cuerpo y el entorno, ya que el espacio por el cual el otro surge, depende del movimiento e integración del cuerpo que crea espacio. Tanto las intenciones, los deseos y las creencias son comportamientos corporales esparcidos. De esta idea se desprende que el otro es a su vez una resonancia del encuentro del cuerpo viviente y de la subjetividad del cuerpo vivido. El cuerpo aquí es el trasfondo movible en constante transformación y conservación en que cada evento orientado a la idea de mundo posee un suelo afectivo que le otorga continuidad y sentido a la percepción.

“El sentir es esta comunicación vital con el mundo que nos lo hace presente como lugar familiar de nuestra vida" (Merleau-Ponty 1975 71). Se infiere entonces que el otro está siempre ligado al horizonte de significación, en tanto cuerpo, ve o toca el mundo desde un nosotros que no puede ser visto ni tocado, por lo cual, jamás será un objeto resuelto para la interioridad o la exterioridad, ya que nunca está completamente constituido. La coexistencia del otro, como un otro ante mí, depende del espacio que inunda la percepción, ya que es a partir del contorno de mi cuerpo que se dibuja la alteridad de lo otro. Lo visible dispuesto a los ojos y lo vidente presentado por el espíritu sintetizan la circularidad de respectividades que se resiste a la idea de un ahí rotundo. Por lo tanto, ¿Cuál es el ahí del otro? ¿Cómo irrumpe ese otro? ¿De qué manera varía la existencia durante la irrupción del otro? Estas preguntas que se realizan de manera abierta, las tomamos para problematizar a partir de ellas la lectura del otro y del cuerpo desde una filosofía del nacimiento intersubjetiva y corporeizada. Una de las primeras afirmaciones que hay que realizar como tesis, es que el nacimiento no es un evento parcial que consiste solamente en la expulsión de un individuo, sino más bien, corresponde a un proceso histórico que involucra el embarazo y el puerperio.

Nacer no es ser la fundación de uno mismo, sino estar situado tanto en la naturaleza como en la cultura: es poseer una fisiología que no se elige, encontrarse a sí mismo en un contexto sociohistórico que uno no ha establecido" (Merleau-Ponty 1975 347).

En relación a esta cita, se asume que el nacimiento desborda la comprensión unipersonal, por lo tanto, se nos presenta inevitablemente como un proceso histórico y relacional mediado por cambios que se experimentan desde que se forma la vida durante la gestación. He de allí porque es importante considerar el embarazo dentro una reflexión de la filosofía del nacimiento. Considerando que la manera en que uno llega al mundo también está mediada por quienes asisten al encuentro, debido a que la primera aparición está suscrita a una tecnología del 
parto que es cultural. Bajo esta tecnología hay modos y costumbres en la manera que el ser humano debe salir de la placenta, así como también, acerca de cómo los otros que asisten al nacimiento han de recepcionarlo. Considerando lo expuesto, resulta infructuoso pensar una filosofía del nacimiento limitada exclusivamente al "nacimiento" como un ser en vías de constitución y, por ende, como un existente en potencia que marca su presencia bajo la forma de una revelación. Esta idea resulta ineficaz para una comprensión histórica y encarnada del ser humano que se proyecta vidente en la mutabilidad del cuerpo.

La propuesta que esbozamos acerca de una filosofía del nacimiento, que englobe la triada embarazo-nacimiento-puerperio, se funda en la importancia de las etapas en el comienzo de la vida de los seres humanos. En el caso de la filosofía es el lugar desde donde problematizamos la cuestión del nacimiento y en donde se ha desplazado su interés más hacia la muerte en vez que a la vida. Consideramos importante establecer que esta triada embarazo-nacimiento-puerperio como presupuesto de una filosofía del nacimiento, conlleva un trasfondo existencial que contempla espacialidad, temporalidad e historicidad, el cual la sitúa como un fenómeno existencial. Sin embargo, Heidegger (1998) no le otorgó relevancia. Desde una perspectiva intersubjetiva, quienes viven este proceso le otorgan significado a la circunstancia que presiden, así como también, el que nace, a futuro, puede referir a su comienzo en el mundo a partir de los otros (Fink 1996). En el caso de una filosofía del nacimiento intersubjetiva y corporeizada, hay que cautelar que la intersubjetividad vivida como padre, madre e hijo, está sujeta a condiciones particulares que delimitan los tipos y formas de relacionarse, de allí también, variaciones de la existencia.

Una de estas limitaciones es la conformación del grupo de familiar, en la cual se relativiza aquello que podríamos describir como los otros, entiéndase la presencia y ausencia del padre, abuelos y hermanos. La filosofía del nacimiento es siempre desde un nosotros. De esta manera, la reflexión que se presenta a continuación acerca de los otros es sólo una referencia aproximada, ya que sólo consideraremos dos tipos de relación: madre-hijo y madre-padre-hijo en el embarazo y el nacimiento.

\section{El embarazo y nacimiento: Cuerpo e intersubjetividad}

Establecido lo anterior, nos preguntamos primeramente acerca de la relación madre-hijo: ¿Cuál es el comienzo del otro? ¿Cómo irrumpe el otro dentro del otro? ¿De qué manera varía su existencia durante el embarazo? Si tomásemos los planteamientos de Heidegger (1998) acerca de que el otro es en relación al ahí en el cual nos encontramos, ¿cuál es ese ahí en que la madre y el hijo se encuentran? 
¿Cuál es ese ahí como vivencia fáctica donde el otro comparece en el mismo horizonte de ocupación? La respuesta es que no hay ahí, pues no hay un trasfondo cronológico-topográfico trascedente por el cual la madre y su hijo se encuentren haciendo lo mismo. De hecho, en el embarazo no hay un ahí rotundo y menos está abierto a lo público como encuentro fáctico. Se desprende entonces que una lectura del Ser-con-el-otro resulta ineficaz para referir al otro en el marco de una filosofía del nacimiento intersubjetiva donde el otro no comparece en la condición de compartir el mundo, ya que el otro es considerado en vías de desarrollo existencial, ya que aún no tiene conciencia de mundo, por lo cual, no existe y, por ende, no se encuentra en la misma situación que la madre.

Retomando la pregunta ¿cuál es el comienzo del otro? La respuesta está sujeta a diversas situaciones, por ejemplo, el momento en la cual la mujer se asume como madre, pues es desde esta adscripción que se admite la alteridad de su hijo/a. Algunas mujeres lo asumen desde la certeza del hecho biológico que se constata en el test de embarazo, cuya reafirmación afianza la idea de que hay un otro en ella que todavía no es visible, pero que es visible al espíritu. A partir de esto, la madre experimenta cambios en su subjetividad desde que sabe que hay otro allí reverberante en lo subrepticio de la carne. Otras mujeres asumen que son madres cuando escuchan los latidos del corazón, o cuando experimentan los cambios en su panza, tanto expansión, como movimientos internos. El latido del corazón es la certeza sonora de la inmensidad del otro, pues colma todo el espacio con una agitada presencia de un "heme aquí". En el caso de la primera situación, está la idea de que el hijo/a como otredad, se reafirma en el encuentro de la certeza de una presencia que todavía no es para el cuerpo viviente, pero que se presenta disponible como subjetividad del cuerpo vivido. En la segunda situación, se puede establecer que el hijo/a como otredad se presenta en la maleabilidad del cuerpo de la madre, es decir, en su excentricidad, cuya aceptación de que ella misma es un cuerpo, en el cuerpo y fuera del cuerpo, enfrentada a su propia existencia, aferrándose y resistiéndose, pero siempre desde un cuerpo unificado (Plessner 1964). Este tocar desde adentro del otro reafirma la tesis de que la transgresión de los límites del cuerpo de la madre obedece a anunciamiento del hijo/a que, aún no resuelto físicamente, se delata desde ella y a través de ella, para sí y para otros.

En relación a lo expuesto anteriormente, y tomando una frase de la cita del poema La Materia es mi Madre de Gonzalo Rojas presentada al inicio de este texto acerca de "cuánto es el gran latido de su prudencia, qué congoja la estremece al tocarme por adentro", es inevitable no pensar en una filosofía del nacimiento intersubjetiva, ya que en la excentricidad de la madre, cuyo latido del hijo/a como otro la confronta consigo mismo desde su propia corporalidad, le provoca una congoja que delata e irradia a los otros que participan del evento, transformando 
también así sus cuerpos y por ende, sus existencias. Es así como la madre y su hijo, lo uno y lo múltiple, se reafirman desde un cuerpo para el cuerpo que está en continua transformación y actualización.

Ante la pregunta ¿cómo irrumpe el hijo/a como otro en el embarazo de la madre y el padre?, se puede responder a partir de la transgresión de sus propios límites del cuerpo, los cuales, se experimentan desde el exceso de lo disponible. En este sentido, los cambios que se experimentan desde el cuerpo viviente como el ensanchamiento de la panza, la palidez de la piel, la turgencia de los senos, están en plena resonancia sobre las transformaciones del cuerpo vivido. Aquí la subjetividad de la madre encarna una serie de mutaciones respecto a su autopercepción y la percepción de su hijo/a, así como también, la relación con los otros. La sinuosidad auspiciosa que va exhibiendo el cuerpo de la madre en función del crecimiento de su hijo/a es la confirmación palpable al tacto y los ojos, y más allá de ellos, pero a través de ellos y con ellos, que hay otro que la desborda, que asiste tras y sobre el velo de carne al encuentro con otros. Esta reafirmación de la existencia que se da en la coexistencia se manifiesta en la maleabilidad del cuerpo. La mutabilidad del cuerpo de la madre es la morada del acontecimiento para un yo-otro que nunca deja de cesar, pues cuyo hijo/a irrumpe como otro en la certeza del golpe. El movimiento es la confirmación del ser, pero no en un ahí como si alguien estuviese disponible y total que se anuncia determinado, sino más bien, en la fuga del ahí, en aquello que está siendo y dejando de ser.

Alrededor del cuerpo percibido se forma un torbellino en el que mi mundo es como atraído y como aspirado: en esta medida, no es ya sólo mío, no me es ya solamente presente, es presente a $\mathrm{X}$, a esta otra conducta que empieza a dibujarse en él (Merleau-Ponty 1975 365).

Este torbellino del cuerpo percibido no le es de suyo a la madre o de suyo al hijo/a, sino que a un nosotros que es mediado por un horizonte de significación por el cual la percepción maleable del cuerpo resulta un testimonio de un yo que es excedido por un otro que comparece en el plano de la actualización del viviente como un yo-otro. Este otro no es interioridad como un ser-con-el-otro u otredad independiente de la percepción determinada como ser-es-exterioridad. Aunque este otro toca desde adentro y se percibe en la superficie de la panza, ambos aspectos resultan ser interdependientes impulsados por una energía circular. Más enfático aún, el horizonte interior de un otro no puede devenir otro sin que los objetos circundantes devengan horizonte, ya que la percepción es un acto con dos caras (Merleau-Ponty 1975). La irrupción del otro se manifiesta en la maleabilidad de la curvatura de la panza de la madre que ampara el estallido de su presencia.

Asimismo, el padre contempla al hijo/a en la agitación de su presencia que jala desde adentro, ve, toca la deformación de la superficie de la panza de la 
madre, en la cual se prolonga la expectativa acerca del misterio de ese ser que se delata. El padre como testigo de la revelación percibe a través del tacto y la visión la presencia de su hijo/a como otro. La temporalidad por la cual el otro se anuncia es co-dependiente de la evolución de la gestación. El tiempo del otro es el tiempo del nosotros, madre, padre e hijo, de manera que cambian sus existencias, pues cambia su percepción respecto al estado que se presenta en un espacio vital compartido. El milagro de la vida es el milagro del cuerpo de un nosotros, de allí que el otro no es nunca otro siendo ahí, sino más bien, otro que está siendo y dejando ser desde una ontología de lo inédito. Por otra parte, y considerando la propuesta teórica de Lévinas (1995) acerca del otro como ser es exterioridad, es menester preguntarse ¿cuál es el otro en el embarazo utilizando la categoría del rostro como fijación del infinito? Considerando que Lévinas (1995) sostiene que el movimiento del rostro, la posición de la cara, es parte del otro que difiere de sí, se puede inferir que en la filosofía del nacimiento intersubjetiva leída desde Lévinas (1995) no habría un otro, ya que ¿cuál es ese rostro del otro durante la gestación que fija la negación del yo? Según Lévinas (1995) "la alteridad que se expresa en el rostro provee la única materia posible a la negación total" (213).

En Lévinas (1995), el otro es siempre otro fuera del espacio del embarazo que ya está constituido como existente dotado de expresión. En este sentido, "el otro permanece infinitamente trascendente, infinitamente extranjero, pero el rostro en el que se produce su epifanía y que me llama, rompe con el mundo que puede sernos común" (208). La cita anterior confirma que no hay intersubjetividad. Si meditásemos acerca del rostro del otro en gestación, debiese asumirse que el rostro es por determinación biológica un cuerpo en revelación, por lo tanto, adscrito a una ontología de lo inédito que otorga textura de lo visible en la constitución de lo vidente. Tal afirmación se sustenta primero en la tecnología de la ecografía por la cual el rostro ha de ser proyectado en el cosmos digital del pixelaje que contribuye a la definición de la forma. Por otra parte, está la posición del bebé que ha de permitir exhibir su rostro. Finalmente, y partir de la ecografía, el rostro se coconstituye en el contraste de la imagen de los padres, es decir, con y a través de la percepción conjunta. En este sentido, el consenso intersubjetivo entre el hecho biológico manifiesto en la resolución gráfica y la proyección social de la imagen del rostro, constituyen la alteridad del otro.

Entre mi consciencia y mi cuerpo tal como lo vivo, entre este cuerpo fenomenal y el del otro, cual lo veo desde el exterior, existe una relación interna que pone de manifiesto al otro como consumación del sistema" (Merleau-Ponty 1975 364).

Ese otro que se manifiesta desde la circularidad de la percepción por el cual el cuerpo de él se anuncia vivido al espacio de mí significación sensorial, confirma a 
través del movimiento el ser del otro. En el caso del nacimiento, el cuerpo del hijo/a como otro, adquiere la condición de cuerpo auspicioso en la expectativa de los otros que están a la espera. Los otros como testigos del cuerpo auspicioso que se anuncia conlleva al sentimiento límite de la paradoja del inicio y el final. El otro deja de ser lo que puede ser para finalmente ser aquello que es en tanto la percepción intersubjetiva de quienes participan de su llegada. Con lo anterior, no se quiere decir que el otro llega a ser, pues ya es, pero dado a que es posible solo con y a través de la percepción, se actualiza su existencia y también la de los que comparecen a su presencia.

El nacimiento está marcado por el cuerpo que explota, ya que el cuerpo de la madre comienza a experimentar una sumatoria de dolores que la preparan para el parto. Entre estos se presenta la sensación que las caderas se ensanchan y la contracción del músculo uterino por la cual las fibras se endurecen. Estas contracciones de la madre son la certeza fisiológica de la arremetida inminente de la revelación. La ruptura de la placenta es la confirmación del tiempo que anuncia el origen, que sumada a la fuerza oculta de la matriz uterina expulsa al otro y lo deja en la circunstancia de arrojo y apertura de un yo-otro. Una vez que el bebé es sacado, el llanto es el modo por el cual, la madre, el padre y el hijo se descifran como otros desde un nosotros constituyéndose en y por la percepción.

\section{Conclusiones}

Considerando la reflexión filosófica presentada acerca del nacimiento como fenómeno intersubjetivo y corporeizado, se pudo establecer una aproximación original a la comprensión del otro durante el embarazo y el nacimiento que se diferencia de nociones escasas y clásicas sobre éste. Para esto se expusieron a modo de contextualización las propuestas acerca de la comprensión de lo otro presentadas por Heidegger (1998) en la coexistencia y la noción de otredad propuesta por Lévinas (1995a) como relación ética basada en la tesis de que el ser es exterioridad. Luego, y a modo de exponer nuestra propuesta de filosofía del nacimiento se tomaron en consideración los planteamientos de Merleau-Ponty (1975) en el capítulo del "Cuerpo y el otro" correspondiente a la Fenomenología de la Percepción. A partir de esta lectura reflexiva se enunciaron dos tesis para sustentar la propuesta de una filosofía del nacimiento intersubjetiva y corporeizada. La primera consistió en que la filosofía del nacimiento debe incorporar tres etapas fundamentales como es el caso del embarazo, el nacimiento y el puerperio. En relación a la segunda, se planteó que el embarazo y el nacimiento es una vivencia de un nosotros, es decir, madre-padre-hijo/a. Ambas tesis se fueron desarrollando en contraste con los planteamientos de Heidegger y Lévinas, en las cuales se deja 
entrever limitaciones importantes para comprender el otro desde una filosofía del nacimiento.

Estas limitaciones se enmarcaron en que los planteamientos de Heidegger obedecen a una concepción de ontología existencial donde la coexistencia comparece en el ahí fáctico por el cual el otro se encuentra en la misma situación que yo vivo, por lo cual, el ser del otro es interioridad. Respecto al análisis del Otro de Lévinas como superación del sujeto trascendental y de que el ser es interioridad, consideramos problemático la lectura de la alteridad en la idea del Rostro como resonancia ética del ser como exterioridad, ya que el otro es codependiente del cuerpo, de manera que no puede imponerse como una categoría de fijación del infinito del otro fuera de la acción de la percepción y la construcción relacional de éste en las etapas de gestación. Finalmente, planteamos que la comprensión del otro en una filosofía del nacimiento intersubjetiva y corporeizada, permite profundizar y otorgar lucidez acerca desde una concepción del Nosotros en el en-sí. De manera que el otro en el embarazo y el nacimiento, no está encerrado en la perspectiva de la madre o del padre, ya que esta perspectiva no posee unos límites definidos o correlatos exclusivamente biológicos ofrecidos por el cuerpo viviente, sino también, incorpora la subjetividad del cuerpo vivido, debido a que espontáneamente se desliza en percepción la del otro y ambas son conjuntamente recogidas en un solo mundo en el que todos participan como sujetos anónimos de la percepción que transforman sus existencias.

\section{Bibliografía}

Arendt, Hannah. La condición humana. Traducido por Ramón Gil Novales. Buenos Aires: Paidós, 1993.

Aurenque, Diana y Jaran, François. "La enfermedad como rasgo humano. Hacia una consideración de la enfermedad en cuanto fenómeno existencial". Revista Alpha 47 (2018): 161-176.

Díez, Fernando. “La filosofía y los nacidos. ¿Por qué los filósofos olvidaron el nacimiento?". Revista de Filosofía Franciscanum 157 (2012).

Fink, Eugen. Oasis de la felicidad. México: UNAM, 1996.

Heidegger, Martín. Ser y tiempo, trad. Jorge E. Rivera. Santiago: Universitaria, 1997.

Johnson, Felipe. "La investigación acerca del otro y la filosofía existencial". Revista Actuel Marx/ Intervenciones 12 (2012).

Lévinas, Emmanuel. El tiempo y el otro. Barcelona: Editorial Paidós, 1993. 
Lévinas, Emmanuel. Totalidad e infinito: ensayo sobre la exterioridad. Salamanca: Sígueme, 1995a.

Lévinas, Emmanuel. De otro modo que ser, o más allá de la esencia. Salamanca: Sígueme, 1995b.

Merleau-Ponty, Maurice. La fenomenología de la percepción. Barcelona: Ediciones Península, 1975.

Plessner, Helmuth. “On Human Expression”. In E. Straus, ed., Phenomenology: Pure and Applied: The First Lexington Conference. Pittsburgh: Duquesne University Press, 1964: 63-74. 